\title{
MAXIMUM ACCELERATION IN TWO-DIMENSIONAL STEADY FLOWS OF AN IDEAL FLUID*
}

By CHIA-SHUN YIH (Iowa Institute of Hydraulic Research)

It is well known** that, in a domain free from singularities, the maximum speed in irrotational flows of an incompressible fluid occurs on the boundary of that domain. In this note, it will be proved that for two-dimensional irrotational flows of an incompressible fluid, the maximum magnitude of the acceleration in any singularity-free domain $D$ must also occur on its boundary provided the flows are steady.

The acceleration components in the $x$ - and $y$-directions are, respectively,

$$
\begin{aligned}
& b=u u_{x}+v u_{y}, \\
& c=u v_{x}+v v_{y},
\end{aligned}
$$

in which $u$ and $v$ are the $x$ - and $y$-components of the velocity, and the subscripts denote partial differentiation. The equation of continuity is

$$
u_{x}+v_{y}=0
$$

and the equation expressing irrotationality is

$$
u_{\nu}-v_{x}=0 \text {. }
$$

From Eqs. (1) to (4) it immediately follows that the square of the magnitude of the acceleration is given by

in which $q^{2}=u^{2}+v^{2}$.

$$
a^{2}=b^{2}+c^{2}=q^{2}\left(u_{x}^{2}+u_{y}^{2}\right),
$$

If $\phi$ and $\psi$ are respectively the velocity potential and the stream function of the given flow, the complex potential for that flow is

$$
w(z)=\phi(x, y)+i \psi(x, y),
$$

in which $w(z)$ is an analytic function free from singularities in the domain $D$. The wellknown relation concerning the magnitude of the velocity is

$$
q^{2}=w^{\prime}\left(w^{\prime}\right)^{*},
$$

in which the asterisk denotes the complex conjugate, and the primes denote ordinary differentiation. Since $w(z)$ is analytic in $D$, its derivative

$$
w^{\prime}(z)=-u+i v
$$

is also analytic in $D$ and represents a potential flow with velocity components $u_{x}$ and $u_{y}$, concerning which the result analogous to (6) is

$$
u_{x}^{2}+u_{\nu}^{2}=w^{\prime \prime}\left(w^{\prime \prime}\right)^{*} .
$$

From (5) to (7), it follows that

$$
a^{2}=w^{\prime} w^{\prime \prime}\left(w^{\prime} w^{\prime \prime}\right)^{*}=\left({w^{\prime}}^{2} / 2\right)^{\prime}\left[\left({w^{\prime}}^{2} / 2\right)^{\prime}\right]^{*}=q_{1}^{2}
$$

${ }^{*}$ Received Nov. 1, 1954. This work is supported by the Office of Naval Research under Contract No. N8onr-500.

**H. Lamb, Hydrodynamics, Dover, New York, 1945, p. 39 and p. 47. 
in which $q_{1}$ is the velocity magnitude in the irrotational flow corresponding to the new complex potential $w^{\prime 2} / 2$. But it has been proved that the maximum value of $q_{1}^{2}$ in $D$ occurs on its boundary, hence the same is true for $a^{2}$.

Since $w^{\prime} w^{\prime \prime}$ is analytic in $D$, the result for the acceleration could have been reached through

$$
a^{2}=w^{\prime} w^{\prime \prime}\left(w^{\prime} w^{\prime \prime}\right)^{*}
$$

in (8) by means of the well-known maximum-modulus theorem for analytic functions, without resorting to $q_{1}$. However, since the corresponding result for the speed is often reached without the use of the maximum-modulus theorem, the present proof may seem preferable to some.

As a by-product of the proof, a means of evaluating the magnitude of the acceleration at any point in the field of flow is provided by (8), once $w(z)$ is known. Furthermore, since the acceleration is proportional to the gradient of the dynamic pressure, it follows from the present result that the maximum dynamic-pressure gradient in $D$ must occur on its boundary.

The boundary of $D$ is, of course, not necessarily a solid boundary. However, for ambiently uniform flows past arbitrarily shaped bodies, the maximum acceleration (or the maximum dynamic-pressure gradient) must occur on one of the solid boundaries. This can be seen by taking $D$ as the entire domain outside the solid bodies. Its boundary then consists of the solid boundaries and a boundary at infinity. Since the acceleration at infinity is zero, the maximum acceleration must occur on one of the solid boundaries.

\title{
AN ESTIMATE OF THE ERROR DUE TO THE TRUNCATED BOUNDARY IN THE NUMERICAL SOLUTION OF THE BLASIUS EQUATION
}

\author{
By L. A. RUBEL' (U. S. Naval Proving Ground*, Dahlgren, Va.)
}

We seek a numerical solution of the Blasius differential equation:

$$
f^{\prime \prime \prime}(x)+f(x) f^{\prime \prime}(x)=0
$$

satisfying the split conditions

$$
0=f(0)=f^{\prime}(0), \quad f^{\prime}(\infty)=2 .
$$

The condition $f^{\prime}(\infty)=2$ is clearly unsuited for numerical procedures. In previous tabulations (e.g. [1], [2], [3]), the condition $f^{\prime}(\infty)=2$ is replaced by $f^{\prime}(M)=2$ where $M$ is chosen so large that the second derivative becomes indistinguishable from zero, and further integration becomes impossible. That this procedure yields, in any sense, an approximation to the desired function has not, to the author's knowledge, been established heretofore.

This paper demonstrates that for large $M$, the numerical solution lies close to the true solution, gives a computable estimate of the deviation from the true solution, and provides an a priori criterion by which any desired accuracy may be achieved.

${ }^{1}$ Received Nov. 12, 1954. The author is now at Cornell University.

*The opinions and assertions expressed herein are the private ones of the writer and are not to be construed as official or reflecting the views of the Navy Department or the Naval Establishment at large. 